\title{
La segregación por género en la Formación Profesional (FP). Hallazgos y propuestas a partir de un proceso participativo de diseño de políticas públicas de juventud en Ontinyent, Valencia, España
}

\author{
Gender segregation in Vocational Training (VT). Findings and Proposals \\ from a Participatory Process of Designing Youth Public Policies \\ in Ontinyent, Valencia, Spain
}

Javier Ortega, Danny Piciucchi, Raúl Ruiz-Callado y Pablo de Gracia-Soriano'

\begin{abstract}
Resumen
La Formación Profesional (FP) es uno de los campos sobre los que más debate está habiendo en la actualidad. Se trata de una modalidad formativa vinculada expresamente al mercado laboral y que tiene, por tanto, efectos sustanciales en el desarrollo profesional de la juventud. Este artículo pretende ofrecer reflexiones y propuestas que surgen de la juventud que participó en el proceso de investigación y en el diseño del I Plan de Juventud de Ontinyent (Valencia, España). En concreto, se presenta la Formación Profesional desde una perspectiva de género y desde la mirada de la juventud, con un enfoque participativo que permite la reflexión, el debate, la deliberación y el diseño bottom-up de ejes estratégicos para la ejecución de políticas públicas. A partir del consenso en la existencia de un escenario actual con una clara segregación por género en la FP, se ponen en relación tres ideas-fuerza sobre las cuáles se profundiza, desde un marco cualitativo, en las causas, los efectos y las posibles propuestas que deberían programarse para revertir dicha problemática. Estas ideas-fuerza son: la estigmatización de la FP, la segregación por género en la FP en función de las familias profesionales y los procesos de orientación formativa. En definitiva, se presenta una investigación que atiende a nuevas tendencias emergentes en este campo, tratando al mismo tiempo de contribuir a valorar la FP, resituar el debate desde la perspectiva de género y, además, visibilizar las potencialidades de los procesos participativos en el marco del diseño de políticas públicas de juventud.
\end{abstract}

\section{Palabras clave}

Segregación, género, formación profesional, políticas públicas, participación, juventud.

\section{Abstract}

Vocational Training (VT) is one of the fields on which more debate is currently taking place. It is a training modality expressly linked to the labor market and, therefore, has substantial effects on the professional development of youth. This article aims to offer reflections and proposals that arise from the youth who participated in the research process and in the design of the I Youth Plan of Ontinyent (Valencia, Spain). Specifically, Vocational Training is presented from a gender and youth perspective, with a participatory approach that allows reflection, debate, deliberation and bottom-up design of strategic axes for the execution of public policies. Based on the consensus on the existence of a current scenario with a clear gender segregation in VT, three force ideas are put into relation on which, from a qualitative framework, the causes, effects and possible proposals that should be programmed to reverse this problem. These force ideas are: stigmatization of VT, gender segregation in VT based on professional families, and formative orientation processes. In short, an investigation is presented that addresses new emerging trends in this field, while trying to contribute to valuing VT, resituting the debate from a gender perspective and, in addition, making visible the potential of participatory processes within the framework. of youth public policy design.

\section{Keywords}

Segregation, gender, vocational training, public policies, participation, youth.

\section{Cómo citar/Citation}

Ortega, Javier; Piciucchi, Danny; Ruiz-Callado, Raúl y de-Gracia-Soriano, Pablo (2020). La segregación por género en la Formación Profesional (FP). Hallazgos y propuestas a partir de un proceso participativo de diseño de políticas públicas de juventud en Ontinyent, Valencia, España. Revista de Sociología de la Educación-RASE, 13 (3), 328-345. http://dx.doi.org/10.7203/RASE.13.3.16588.

\footnotetext{
1 Javier Ortega, Universidad de Alicante, j.ortega@ua.es; Danny Piciucchi, Universidad de Alicante, dp44@gcloud.ua.es; Raúl Ruiz-Callado, Universidad de Alicante, raulruiz@ua.es; Pablo de-Gracia-Soriano, Universidad Complutense de Madrid y Universidad de Alicante, pablodeg@ucm.es.
} 


\section{La formación profesional en el contexto de la desigualdad de género}

La Formación Profesional es una opción cada vez más demanda por el mercado laboral en España. Además, el actual tejido productivo tiene serias dificultades para absorber a todos los egresados universitarios y cada vez se demandan más puesto técnicos intermedios con formación especializada. La evolución de la FP en España ha ido en paralelo a la progresiva masificación de las universidades españolas, por lo que se ha consolidado un modelo de primera y otro de segunda categoría. El desarrollo y consolidación de ambas opciones formativas determinará la sociedad de los próximos años. Otro de los efectos palpables tiene relación con el papel de las mujeres en los grados de FP, ¿tienen la misma presencia que los hombres?, ¿se crean ramas profesionales mixtas con una participación similar de hombres y mujeres? Aunque no sean dos interrogantes con una elevada carga teórica-conceptual, son de gran utilidad para invitar a la sociedad civil a que adopte una posición crítica al respecto. De preguntas sencillas derivan respuestas complejas.

En el marco de los estudios sobre el mercado laboral y el sistema educativo, la Formación Profesional (FP) se presenta como una dimensión analítica determinante para la sociedad actual. Entorno a la FP se están originando diferentes debates que suscitan un gran interés entre la comunidad investigadora, véase, por ejemplo, la tendencia de la preferencia de la universidad frente a la FP y cómo esta situación está generando un desequilibrio en la demanda laboral; también cabe destacar el asunto de la escasa adaptación de la FP a las necesidades del tejido productivo (Merino, 2012). Sin embargo, lo que se expondrá de aquí en adelante parte de la incorporación del enfoque de género a la situación actual de la FP (Fernández e Ibañez, 2018; Mariño y Rial, 2019; Mosteiro y Porto, 2017; Rial, Mariño y Rego, 2011; Rosado, 2012), en concreto se incluirá un breve corpus analítico que reflexione acerca de la segregación por género en los ciclos formativo de FP.

Pese a la (in)definición global del término de Formación Profesional, tal y como apunta Merino (2012: 504), y sin ánimo de profundizar en aspectos semánticos, se atenderá a lo que la legislación española entiende como FP reglada inicial. Por otro lado, la categoría género es imprescindible si existe la pretensión de profundizar en la temática que nos ocupa y, especialmente, si se busca desenterrar las discriminaciones latentes e invisibilizadas desde una esfera sistémica (Subirats, 2016). Ya en los años 80, este enfoque empezó a tener relevancia significativa en el campo de las ciencias sociales y sirvió, fundamentalmente, para colocar la desigualdad de género en el terreno de lo cultural e histórico y, de este modo, relegar las diferencias biológicas a una posición residual (Rosado, 2012). Incluso desde organismos supraestatales como las Naciones Unidas nos recuerdan que la centralidad del género es fundamental en cualquier faceta de los derechos humanos (Venegas, 2010). Se parte, por tanto, de la premisa de que el género es la «representación cultural, que contiene ideas, prejuicios, valores, interpretaciones, normas, deberes, mandatos y prohibiciones sobre la vida de las mujeres y de los hombres» (Colás y Villaciervos, 2007: 37), teniendo, por tanto, efectos directos tanto en la esfera privada como en la pública.

A pesar de que el tema de la segregación por género en la FP no ha sido investigado con la suficiente profundidad (Mariño y Rial, 2019: 253), sí que se pueden consultar diferentes informes, estudios o investigaciones que confirman esta problemática (Rial, Mariño y Lego, 2011; Termes, 2012; Mariño y Rial, 2019). Tal y como concluyen algunos informes internacionales ${ }^{2}$, la segregación sexual en el acceso a las diferentes familias profesionales es un hecho generalizado, aunque en determinados países del sur de Europa, como es el caso de España, «crece y persiste una fuerte influencia de una cultura social dominante

2 Se hace alusión a informes presentados por la OIT (1998, 2012); CCOO (2012, 2013); ONU (2012); LANBIDE-CAE (2012). Ver en Mariño y Rial (2019: 253). 
que delimita y condiciona las opciones y las decisiones de la juventud femenina de forma sexista. Podemos decir que la elección de los estudios en la mujer sigue estando condicionada por prejuicios sociales» (Mariño y Rial, 2019: 253).

Tal y como apunta Termes (2012) en su investigación, se distinguen dos modalidades de FP: por un lado estarían los ciclos formativos masculinizados, vinculados de forma directa o indirecta a sectores donde prevalece la mano de obra masculina. Estos pertenecerían a las ramas de instalación y mantenimiento, transporte y mantenimiento de vehículos, edificación y obra civil, informática y fabricación mecánica, entre otras; por otro lado, aunque con una proporcionalidad menor, estaría la FP mayoritariamente feminizada, por ejemplo, por citar algunos de ellas: comercio, administración y gestión, sanidad, servicios a la comunidad e imagen personal (Termes, 2012; Merino, 2012). Algunos investigadores sostienen que debemos relativizar la problemática del sexismo en el actual contexto de la FP, sobre todo en las etapas del primer ciclo, ya que todavía nos encontramos en la fase de (des)estigmatización de dicha alternativa formativa y, por tanto, debemos evitar medidas susceptibles de excluir a muchos jóvenes de la FP (Merino, 2012: 510). No obstante, otras expertas, en contraposición, lo diagnostican como un factor sustancial que refuerza las desigualdades de género en el mercado laboral.

\section{«Esta realidad de no igualdad se traspasa al plano laboral, donde siguen creciendo la brecha salarial, la brecha en el emprendimiento y el acceso al empleo, la feminización o la masculinización de sectores profesionales tradicionales y un escaso reconocimiento a la formación y a los niveles de cualificación alcanzados por las mujeres trabajadoras». (Mariño y Rial, 2019: 257).}

Para ahondar en las causas del mismo se ha de partir de un esquema analítico que interprete los procesos de socialización desde un enfoque de género y reconocer que la educación es una parte sustancial que va «desde los roles que marcan la vida familiar a las imágenes que impregnan todo lo que les rodea en su vida diaria, en los cuentos y lecturas, en los juguetes, en los libros de texto, en la publicidad, etc.» (Fernández e Ibáñez, 2018: 118). Se configura, desde edades tempranas, una identidad de género determinada que influye categóricamente en el desarrollo educativo y profesional de los varones y las mujeres (Sainz, 2007; Mosteiro y Porto, 2017; Subirats, 2016; Fernández e Ibáñez, 2018), transfiriéndose tanto en el currículum oculto como en el currículum explícito (Mariños y Rial, 2019: 258). Algunos informes apuntan, incluso, que es desde la enseñanza primaria cuando los niños empiezan a discriminar las asignaturas que son más «masculinas» o «femeninas» (Sainz, 2007: 157-158). Este escenario explicaría, a priori, el modelo de expectativa-valor de logro y de elección académica que trabajó Eccles (2001), donde esta última, la elección académica, viene determinada por la creencia de haber adquirido competencias en una determinada actividad unida al valor que se le asigna a dicha tarea. Por consiguiente, se constituye, mayoritariamente entre las mujeres, la pérdida de confianza y de interés en ocupaciones que tradicionalmente se les ha sido asignada a los hombres (Fernández e Ibáñez, 2018). No obstante, diferentes investigaciones concluyen que hay una tendencia progresiva, entre el alumnado de FP, hacia una mayor sensibilización hacia la igualdad (Mosteiro y Porto, 2017: 162). Como veremos a continuación, este proceso de (re) definición de los imaginarios juveniles va en consonancia con el desarrollo de nuevas políticas públicas pensadas desde y para la juventud. 


\section{Políticas públicas y juventud}

Según Lascoumes y Le Galès, «en las sociedades contemporáneas, las políticas públicas están omnipresentes, trátese de asuntos viejos (vagabundaje, armamento), modernos (desempleo, salud) o emergentes (nanotecnologías, regulación de mercados financieros)» (2014: 11). Antes de referirnos, específicamente, a las políticas públicas de juventud y, por ende, focalizar teóricamente nuestro objeto de estudio, haremos una breve alusión a qué entendemos por «políticas públicas»y, sobre todo, subrayaremos las potencialidades de dicha categoría en el contexto que nos ocupa.

Velásquez (2009) enumera hasta veintinueve las encontradas en los más conocidos textos concernientes a las políticas públicas. Sin embargo, a continuación, se enunciarán cuatro definiciones (Dye, 1978; Anderson, 1984; Azzone y Dente, 1999) que aquí son suficientes para identificar qué se entiende por políticas públicas. Según Thomas R. Dye, una política pública es «todo lo que los gobiernos deciden hacer o no hacer» (1978: 3)..

Por su parte, James Anderson afirma que una política pública es «un curso de acción intencional y relativamente estable seguido por un actor o un conjunto de actores para tratar un problema o una cuestión de interés» (1984: 2). Por último, Azzone y Dente (1999), sostienen que una política pública es un conjunto de acciones emprendidas por una pluralidad de actores con el fin de solventar un problema colectivo. Se trata de una serie de actividades inducidas por unas necesidades y/o demandas comunitarias para cuya satisfacción se considera necesaria, en un momento histórico y en un contexto dados, la intervención de las instituciones públicas (Lascoumes y Le Galès, 2014).

En lo que atañe a la juventud, ésta se ha interpretado, durante mucho tiempo, como un periodo de transición cuya razón de ser es dejar de existir como tal para dar paso a la vida adulta (Benedicto y Morán, 2003). Esto ha fomentado la consideración de los jóvenes como ciudadanos incompletos, debido a su situación de múltiples dependencias: sin un empleo, sin una formación acabada o sin una familia propia, por ejemplo. No obstante, en los últimos años ha venido afirmándose un nuevo paradigma, llamado juvenilista, que reconoce a la juventud un estatuto de autonomía oponiéndose al paradigma adultocrático, que ha sido puesto en entredicho.

Las políticas públicas de juventud llevan décadas oscilando teóricamente -pero amalgamándose en la realidad cotidiana- en un continum que va desde políticas de transición hasta políticas de afirmación. Las primeras definen a la juventud desde la adultez y, por consiguiente, se centran en agilizar la consumación de las transiciones ineludibles para lograr el estatus de adulto: la consecución de un empleo, la adquisición de una vivienda, la formación de una familia y la procreación. Mientras que las segundas, focalizan sus esfuerzos en la afirmación identitaria de los jóvenes (experimentación, creatividad, movilidad, autonomía y participación), entendiendo que las primeras más que políticas de juventud son políticas sociales universales y, por lo tanto, no pueden considerarse específicamente políticas de juventud. En este sentido, cabe añadir el siguiente interrogante: ¿si la formación para el empleo es un proceso por el cual el alumnado transita para incorporarse al mercado laboral (dimensión adulta), por qué se considera pertinente aludir a la «afirmación identitaria de la juventud?». Aunque resulte paradójico, la visión juvenilista no se contrapone a la esfera adulta, más bien forma parte de la misma. El objetivo es, por consiguiente, determinar desde que perspectiva es más provechoso atender a las realidades formativas de la juventud y, sobre todo, ser capaces de promover colectivamente disposiciones creativas e innovadoras para el fomento de una FP atractiva y orientada a los intereses del alumnado. 
En función de la concepción teórica que se adopte acerca de las políticas públicas y de la juventud, se diseñarán e implantarán unas políticas de juventud u otras. De hecho, según Benedicto y Morán (2003), el paradigma adultocrático y el juvenilista que mencionábamos anteriormente, se reflejaron en la práctica en el diseño y en la implementación de políticas públicas de juventud de transición y de afirmación, respectivamente.

Por tanto, en consonancia con la perspectiva juvenilista, que, reiterémoslo, persigue promover la experimentación, la creatividad, la movilidad, la autonomía y la participación juvenil, se ha reparado en que la adopción de un enfoque participativo es la opción metodológica más idónea para contribuir a este nuevo paradigma emergente. En este sentido, asumimos la responsabilidad, como científicos sociales, de ser consecuentes con el nuevo contexto en el que el imaginario juvenil se pone en el centro de las políticas públicas promovidas por los organismos competentes.

\section{Metodología}

\subsection{Fundamentos metodológicos}

Las nuevas lógicas de gestión municipal que algunos califican como «gobernanza participativa local» (Hernández, E., Camacho, R., Silván, A., Rojas-Martín, F. \& Stan, L, 2018) fueron determinantes a la hora de demandar la necesidad de sistematizar, clasificar, ordenar y definir un horizonte a corto-medio plazo en lo que a políticas públicas de juventud se refiere. Hasta la fecha, los propios agentes políticos del municipio sostenían que las decisiones que se dirimían hasta ese momento partían de dos pretextos fundamentales: por un lado, de la identificación cortoplacista de los problemas que acuciaban a la juventud y, en paralelo, de la dependencia cuasi exclusiva del personal técnico de la corporación municipal. En consonancia con la voluntad política se diseñó una propuesta de investigación empírica enmarcada en la Investigación-Acción Participativa (IAP), la participación y la inclusión de los enfoques de género en los procesos de investigación sociológica. Los fundamentos metodológicos que han guiado el proceso de planificación estratégica de políticas municipales de juventud se agrupan en cuatro dimensiones: trabajo técnico-académico, enfoque participativo, perspectiva de género y perspectiva transversal y territorial.

La labor técnica y científica del proceso de planificación ha sido un factor clave en todo el proyecto, especialmente en la fase de sistematización, operacionalización y análisis de la información. La rigurosidad y la planificación metodológica de carácter técnico y científico han servido de baluarte para incorporar otras perspectivas y enfoques al proceso de planificación (de-Gracia-Soriano, Jareño-Ruiz, Jiménez-Delgado, 2019; Carratalá-Puertas, Ruiz-Callado, de-Gracia-Soriano, 2019) y, de este modo, trascender el diseño de políticas públicas desde un marco unidimensional.

Respecto a la segunda dimensión, referente al enfoque participativo, es necesario reseñar que cuando se incluye el término «participativo» en el campo de las ciencias sociales, la investigación social y/o el diseño de políticas públicas, se corre el riesgo de ser objeto de la manida pregunta de si se está ante un proceso ciertamente participativo, o si se está ante otra experiencia más de tono pseudo-participativo (Caballero, Martín y Villasante, 2019; Ganuza y Francés, 2012). En este caso, el proyecto parte de un enfoque participativo y debido a su contexto, se encuentra sometido a una serie de limitaciones espacio-temporales y de operacionalidad que no permiten referirse a él como un proceso IAP, stricto sensu. 
La perspectiva de género, que también fundamenta el proceso de planificación estratégica, es un imperativo moral que cobra relevancia cuando se trabaja en el diseño de políticas públicas de juventud (Goig y Nuñez, 2011; López, 2016; Ortiz, 2008). Para revertir el problema estructural de la desigualdad de género no basta con sugerir actuaciones estratégicas que vayan en esta línea; se tiene la obligación de ir un paso más allá y seguir los dictámenes que se están aplicando progresivamente en el conjunto de instituciones, administraciones públicas, universidades, etc., que no es otro que partir de marcos cognitivos, normativos y metodológicos sustentados en la perspectiva de género.

\subsection{Técnicas de investigación y fases del trabajo de campo}

Algunos expertos consideran que las metodologías participativas no son tanto «una metodología como un conjunto de posicionamientos, una serie de métodos aplicados que tienen como fin primordial la transformación social a través de la acción» (Francés et al., 2015: 62). Dicho de otra manera, la IAP más que una técnica, es un «enfoque o estrategia general de análisis e intervención que se puede plasmar en muchas prácticas concretas que dependerán de la creatividad de los protagonistas y de las circunstancias presentes en cada caso» (Colectivo IOE, 2004: 2); es, en definitiva, una cuestión de paradigma y no tanto de técnicas (Caballero, Martín y Villasante, 2019: 27). De hecho, «el abanico de técnicas susceptibles de ser utilizadas dentro de una investigación participativa es verdaderamente amplio y diverso. Ni todas las técnicas sirven para el mismo fin, ni producen el mismo tipo de información con su aplicación» (Francés et al., 2015: 85). Las características del proyecto presentado requieren utilizar diferentes técnicas de investigación que combinan diagnosis y planificación, tal y como puede apreciarse en la Figura 1. En este caso, se ha hecho uso del grupo focal o focus group, la técnica DAFO-CAME, el flujograma, la construcción de matrices de priorización y la configuración de talleres participativos.

Figura 1. Relación entre diseño de políticas públicas y técnicas utilizadas para ello

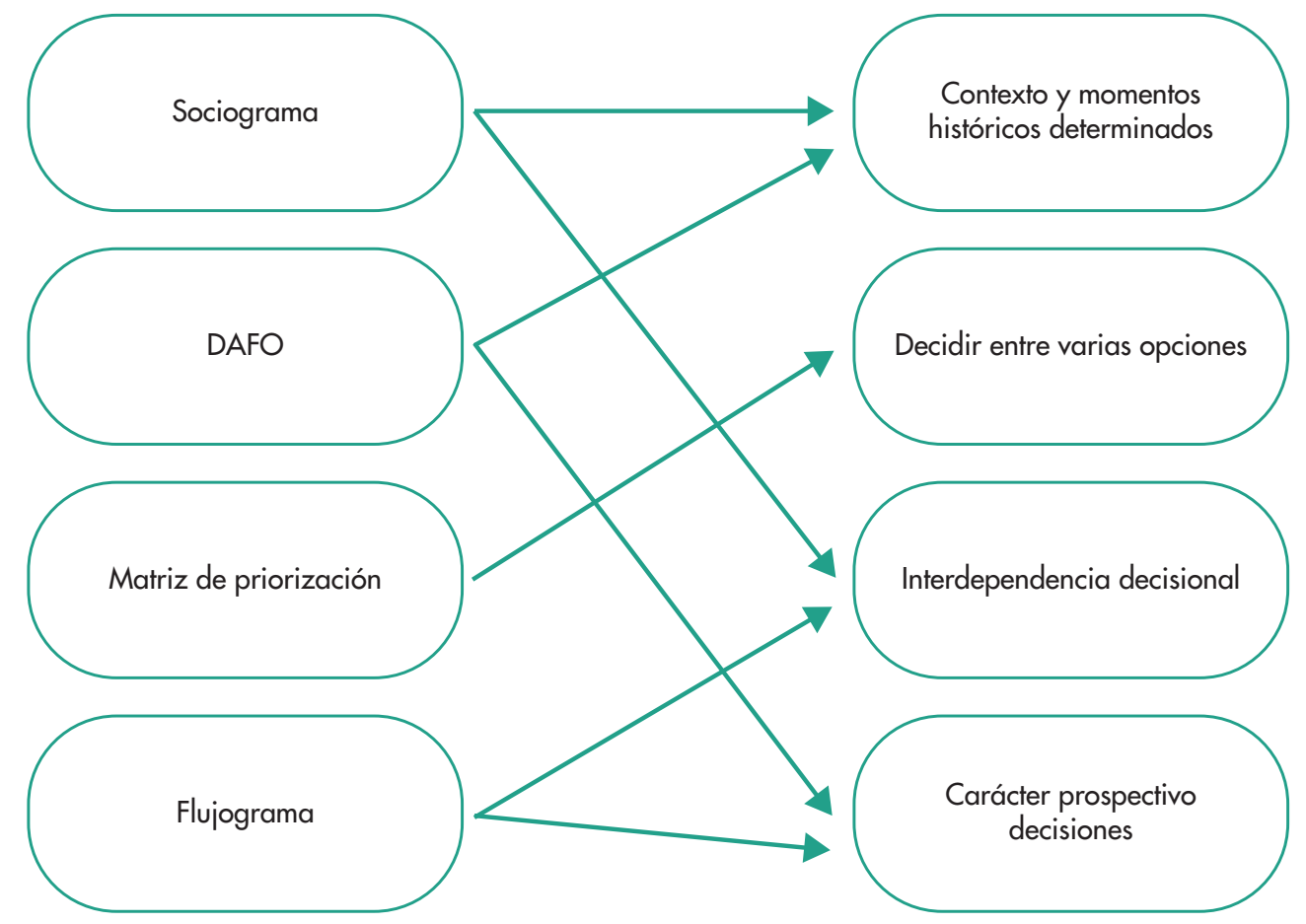

Fuente: Fuente: Piciucchi, 2019. 


\subsubsection{Focus group y DAFO-CAME. Ampliación del enfoque de género}

En el proceso de auto-reflexión del equipo se contempló la necesidad de profundizar en algunas cuestiones que habían quedado relegadas a un plano residual y que, según el criterio técnico, se entendió que debían de incluirse como temáticas centrales en el marco del proceso de planificación estratégica sobre políticas de juventud: la desigualdad de género en la etapa juvenil y adolescente y al generalizado problema de la emancipación tardía de la juventud. En consecuencia, se organizó un grupo focal (GF) en el mes de abril de 2019, donde se seleccionaron a jóvenes del municipio y a profesionales vinculados a las temáticas que son objeto de la investigación.

Las personas participantes tuvieron la oportunidad de debatir, deliberar y consensuar acerca de las diferentes situaciones de desigualdad de género que experimentan las chicas y chicos jóvenes de Ontinyent, al mismo tiempo que realizaban sugerencias para revertir la problemática desde el campo de las competencias municipales. Para dinamizar el taller se aplicó el método del DAFO-CAME (Debilidades, Amenazas, Fortalezas, Oportunidades - Corregir, Afrontar, Mantener, Explotar), a través del cual los y las participantes realizaban aportaciones críticas de forma sistematizada y ordenada (véase la Tabla I), mientras, a continuación, tenían que reformular sus afirmaciones y convertirlas en propuestas. Se trata de un ejercicio de deliberación colectiva donde la puesta en común de argumentos, opiniones y juicios de valor es la auténtica potencialidad de esta técnica de investigación (Francés et al., 2015).

Tabla I. Relación entre diseño de políticas públicas y técnicas utilizadas para ello

\begin{tabular}{lcc}
\hline & INTERNO & EXTERNO \\
\hline Aspectos negativos & DEBILIDADES & AMENAZAS \\
& (Permitirá diseñar Estrategias de Corrección) & (Permitirá diseñar Estrategias de Afrontamiento) \\
\hline \multirow{2}{*}{ Aspectos positivos } & FORTALEZAS & OPORTUNIDADES \\
\hline
\end{tabular}

Fuente: Fuente: elaboración propia a partir de Jareño-Ruiz, de-Gracia-Soriano y Jiménez-Delgado, 2019.

\subsubsection{Talleres participativos: matriz de priorización, flujograma y dinámica de deliberación} Siguiendo la línea del enfoque participativo, se organizaron diversos talleres donde la ciudadanía de Ontinyent, principalmente la juventud, pudo expresar, debatir y discernir colectivamente acerca de los problemas, demandas e inquietudes que afectan a su colectivo. Antes de organizar estos talleres fue necesario partir de un consenso metodológico que estuviera en concordancia con los objetivos del proyecto, tal y como sugieren Francés, Alaminos, Penalva y Santacreu (2015) y Caballero, Martín y Villasante, (2019). En primer lugar, se sugirieron dos principios fundamentales: debía generarse un espacio que incentivara la reflexión colectiva y que se apoyara en el consenso para la toma de decisiones; los procesos de solicitud de propuestas a título individual no se correspondían con la fundamentación metodológica de partida, por este motivo la dimensión cualitativa y deliberativa adquirió una relevancia notable desde el primer instante. En segundo lugar, era preciso integrar a la juventud local en el proceso de elaboración del plan, había que dotarles de un papel pro-activo en la fase de priorización y diseño de los ejes estratégicos que fueran surgiendo. Y, en tercer lugar, se propuso como principio ineludible incluir la perspectiva de género en el diseño y dinamización de los talleres participativos. 
Durante los meses de abril, mayo y junio de 2019 se organizaron en total cuatro Talleres Participativos (TP) dirigidos a diferentes perfiles juveniles, profesionales y técnicos de la ciudad. Partiendo de la necesidad de que participase el mayor número de jóvenes del municipio, el diseño muestral atendió 3 objetivos metodológicos: conseguir una visión heterogénea de las problemáticas que afectan a la juventud (visión técnica, juvenil y adulta), garantizar la presencia de jóvenes en los talleres con una actitud propositiva e implicar a alumnos y alumnas que estuvieran cursando la FP. En total, participaron cincuenta y siete personas, la mayoría jóvenes entre 15-30 años. En términos proporcionales fue similar la presencia de hombres y mujeres, por lo que se pudo analizar la visión de los problemas juveniles desde ambas perspectivas. Los talleres se estructuraron respetando la misma dinámica en todos, aunque el perfil de los participantes varió en función de los objetivos metodológicos. Uno de los talleres estaba dirigido a población adulta, principalmente técnicos del ayuntamiento y representantes de entidades asociativas, en el segundo y el tercero participó dos grupos de estudiantes de dos ciclos de Formación Profesional distintos -un grupo notoriamente masculinizado y otro donde la proporción de mujeres estudiantes era ligeramente superior. Por último, se realizó un taller abierto a toda la ciudadanía en el que asistieron, principalmente, jóvenes entre 20-30 años.

Los talleres participativos se programaron en dos partes. La primera consistía en deliberar y decidir de forma consensuada el grado de prioridad que le otorgaban a una lista de problemáticas asociadas a la juventud del municipio. Trabajaron en grupos reducidos aquellos documentos de priorización de propuestas. Se originó un espacio deliberativo donde se expresaron posiciones críticas muy significativas y se generaron reflexiones y propuestas de gran interés a partir de las aportaciones e intervenciones que se realizaban. En la segunda parte se incorporó un nuevo elemento: el flujograma. Consiste en crear colectivamente una matriz para ubicar las necesidades que han sido señaladas previamente como prioritarias en función del grado de control para revertirlas (eje vertical) e identificar a qué grupo de edad les afectaría preferentemente (eje horizontal). Una vez que completaron este primer paso, los participantes tuvieron que interrelacionar el conjunto de necesidades mediante flechas unidireccionales siguiendo la lógica causa-consecuencia. El flujograma es una técnica que permite visualizar las relaciones de causa-efecto entre los diversos elementos relacionados con el tema objeto de debate y los actores o agentes implicados, a fin de establecer los «nudos críticos», y los principales factores por los que cabría comenzar a resolver el problema, así como las propuestas de acción ligadas a ellos (Francés et al., 2015: 138). En consecuencia, proporciona un cuadro en el que los elementos están interrelacionados: los que tienen numerosas flechas de salida hacia otros elementos son las causas, los que reciben numerosas flechas son las consecuencias y, por último, los que abundan de ambas flechas son los nudos críticos, es decir, los elementos por dónde empezar posibles acciones futuras. Una de las ventajas de esta técnica es que facilita, por un lado, la proposición de acciones eficaces sobre los elementos que la población controla y, por otro, el sondeo de las posibles acciones futuras que pudieran favorecer la identificación de aquellos elementos que en la actualidad se escapan del control de la ciudadanía. 
Tabla II. Resumen de los Talleres Participativos organizados en Ontinyent

\begin{tabular}{|c|c|c|c|c|}
\hline TALLERES & TÉCNICAS PARTICIPATIVAS & N PARTICIPANTES & EDAD & GÉNERO \\
\hline GRUPO FOCAL & $\begin{array}{l}1^{0}: \text { DAFO (proceso deliberativo) } \\
2^{0}: \text { CAME (fase propositva) } \\
3^{0}: \text { Puesta en común de resultados }\end{array}$ & $\begin{array}{l}12 \text { personas } \\
\text { Perfiles: } \\
\text { - Jóvenes de asociaciones juveniles } \\
\text { - Técnicos de Instituciones Públicas }\end{array}$ & $\begin{array}{l}\text { 20-30 años } \\
40-50 \text { años }\end{array}$ & $=$ \\
\hline jer TALLER PARTIPATIVO & $\begin{array}{l}\text { 10: Priorización en grupo de propuestas } \\
2^{0} \text { : Elaboración del FLUJOGRAMA } \\
3^{0} \text { : Puesta en común de resultados }\end{array}$ & $\begin{array}{l}18 \text { ióvenes } \\
\text { Perfiles: } \\
\text { - Alumnos (mayoría hombres) de FP }\end{array}$ & 16-30 años & 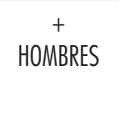 \\
\hline $2^{\circ}$ TALLER PARTIPATIVO & $\begin{array}{l}\text { 10: Priorización en grupo de propuestas } \\
2^{0} \text { : Elaboración del FLUJOGRAMA } \\
3^{0} \text { : Puesta en común de resultados }\end{array}$ & $\begin{array}{l}20 \text { jóvenes } \\
\text { Perfiles: } \\
\text { - Alumnos (mayoría mujeres) de FP }\end{array}$ & 16-30 años & $\stackrel{+}{+}$ \\
\hline 3er TALLER PARTIPATIVO & $\begin{array}{l}\text { 10: Priorización en grupo de propuestas } \\
2^{0} \text { : Elaboración del FLUJOGRAMA } \\
3^{0} \text { : Puesta en común de resultados }\end{array}$ & $\begin{array}{l}7 \text { personas } \\
\text { - Técnicos de áreas del Ayuntamiento } \\
\text { - Representantes de Asociaciones }\end{array}$ & 30-55 años & $\begin{array}{c}+ \\
\text { MUJERES }\end{array}$ \\
\hline $4^{\circ}$ TALLER (GRUPO DE DISCUSIÓN) & $\begin{array}{l}\text { 10: Priorización en grupo de propuestas } \\
2^{0} \text { : FOCUS GROUP }\end{array}$ & $\begin{array}{l}13 \text { jóvenes } \\
\text { - Jóvenes del municipio } \\
\text { - Representantes de asociaciones juveniles } \\
\text { - Técnicos del área de juventud }\end{array}$ & $18-30$ años & $=$ \\
\hline
\end{tabular}

Fuente: elaboración propia.

\section{Principales resultados}

El acceso a la educación obligatoria, así como la posibilidad de disponer de un abanico amplio de oferta formativa son indicadores del desarrollo de una región, y en especial de sus habitantes más jóvenes. En Ontinyent hay once centros educativos en los que se imparte educación primaria y ocho centros de educación secundaria, como queda reflejado en la Tabla II. El bachillerato se imparte en cinco centros, tres de titularidad pública y dos de régimen privado-concertado. El municipio también cuenta con siete centros donde se imparte educación especial, y con tres centros de titularidad pública para cursar Formación Profesional (FP).

La oferta formativa de la FP es relativamente extensa en Ontinyent, tal y como demuestran los diez ciclos formativos de grado medio y siete de grado superior ofrecidos por los centros públicos de enseñanza Jaume I, L’Estació y Pou Clar.

Tabla III. Relación de Grados y Centros Formativos de FP en Ontinyent

\begin{tabular}{ll}
\hline FP GRADO MEDIO & FP GRADO SUPERIOR \\
\hline - Comercio & - Administración de Sistemas Informáticos \\
- Cuidados Auxiliares de Enfermería & - Administración y Finanzas \\
- Emergencias Sanitarias & - Automoción \\
- Explotación de Sistemas Informáticos & - Automoción y Robótica Industria \\
- Gestión Administrativa & - Comercio internacional \\
- Instalaciones de Telecomunicación & - Desarrollo de Aplicaciones Informáticas \\
- Instalaciones Eléctricas y Automáticas & - Higiene Bucodental \\
- Instalaciones frigoríicas y de climatización & \\
- Mantenimiento de Vehículos Autopropulsados & \\
- Mantenimiento electromecánico & \\
\hline
\end{tabular}

Fuente: elaboración propia a partir de los datos consultados en ARGOS y el Diagnóstico Previo del Plan de Juventud. Ayuntamiento de Ontinyent, 2018. 
Los datos ofrecidos por el Censo de Población de 2010 del INE, a propósito del nivel de estudios que tiene la población que reside en Ontinyent, indican que el 14,2\% tiene estudios superiores, cifra notablemente inferior en relación a la media de la provincia de Valencia (19,88\%). En términos históricos, ha habido un claro aumento en Ontinyent de personas que han accedido a la universidad (4,4\% en 1991 y $8,4 \%$ en 2001), tendencia que ha ocurrido de forma similar en el conjunto de la provincia. Respecto a la población que ha alcanzado niveles de estudios medios (educación secundaria, los diferentes grados de FP y el Bachillerato), esta fuente arroja que un $60 \%$ está clasificada en este segmento, cinco puntos más que el mismo dato a nivel provincial.

Sin embargo, estos datos no recogen ciertos discursos y problemáticas que escapan de los análisis cuantificables y que, como se mostrará a continuación, son revelados a través de la aplicación de técnicas cualitativas de investigación. En concreto, este trabajo evidencia tres aspectos que adquieren fuerza en los argumentarios de la juventud, y que por tanto han sido analizados de forma sistemática: la estigmatización de la FP, la segregación por sexo-género de la FP y la necesidad de reformular la orientación socioeducativa en las edades tempranas.

\section{1. 'Los tontitos tienen que hacer FP'. Estigmatización de la Formación Profesional}

A pesar de la variedad de ciclos formativos ofrecidos en Ontinyent, el proceso de investigación revela que el acceso a la FP de la juventud está condicionado por determinados aspectos que dificultan el desarrollo de esta opción como una salida profesional óptima y de calidad, a pesar de las continuas reformas que ha habido en este sector en España (Brunet y Böcker, 2017).

Se ha corroborado en el trabajo de campo, a partir de los grupos focales y los talleres participativos que, en general, la juventud muestra una actitud analítica y crítica respecto a la situación de la formación y el empleo en el municipio. El desarrollo del grupo focal hizo surgir argumentarios que hacen referencia al carácter problemático que tiene para la juventud la toma de decisiones respecto a su futuro en el ámbito educativo, ya que supone pensar, necesariamente, en el mercado de trabajo. En este sentido, los procesos de elección son atravesados por el prestigio social de las opciones de futuro que barajan. Tal como lo plantea Termes (2012), es en los propios institutos de secundaria, ya sea a través de las acciones tutoriales o mediante la orientación educativa al finalizar la ESO, desde donde prevalece la tendencia a establecer la división entre buenos estudiantes -redirigidos a Bachillerato- y malos estudiantes - redirigidos a FP-. En este sentido, ellas y ellos mismos adoptan una posición crítica al respecto señalando que «hay intereses en colocar determinadas plazas en Bachiller y determinadas plazas en FP...» (GF). Las siguientes narrativas muestran, de forma ejemplar, el discurso dominante del grupo:

«Se ha mejorado en la información sobre la FP, pero todavia hay mucho por hacer y debemos tener más información. Se sigue enfocando en que los tontitos tienen que hacer FP, y no es así... está muy estigmatizada la FP». (GF).

"A mi me bubiera gustado que cuando me dijeron que biciera Bacbiller también me dieran la opción de haber hecho una FP, quizá la hubiera hecho». (GF). 
Es necesario recalcar que durante la ejecución de los talleres, realizados en un centro público, también se reveló que, además de la estigmatización por clase intelectual, estrechamente vinculada a la posición socioeconómica de las personas, las y los jóvenes encuentran la existencia de otra norma: la persistencia de prejuicios por cuestión de género, asociados a la rama académica y profesional. Plantean que la elección de los estudios llega a ser determinante en función del sexo que se tiene, más allá de la propia voluntad o el interés.

Por otro lado, y en cualquier caso, entienden que el mercado laboral del municipio no se adapta a las realidades y necesidades formativas de la ciudadanía, por lo que asumen como normal tener que ir a otros lugares para estudiar y/o trabajar. Este contexto les hace reconocer un escenario de incertidumbre respecto a sus trayectorias formativas y profesionales.

\subsection{La segregación de género en la Formación Profesional}

La formación profesional es, cada vez más, la opción preferida por muchos jóvenes para adquirir las competencias formativas necesarias que les valide para desempeñar un trabajo acorde a sus intereses. Aunque la practicidad de algunas opciones de FP esté en cuestionamiento, lo cierto es que la ratio de inserción laboral de los recién titulados es, en líneas generales, sustancialmente elevada.

Además de la estigmatización ligada a la FP persiste otro fenómeno consustancial a la desigualdad de género: la manifiesta segregación que se produce en gran parte de los ciclos formativos de formación profesional que se imparten en los institutos de enseñanza secundaria de Ontinyent: el 64\% de los Grados Medios de FP lo conforma entre el 90\% y 100\% de alumnado masculino, mientras que en el resto las alumnas representan entre el 60\% y el $80 \%$; en lo que respecta a los Grados Superiores de FP, la estructura es similar (en el 75\% de los mismos los chicos oscilan entre el $66 \%$ y $95 \%$ de matriculados, y en los restantes ciclos las mujeres representan entre el $66 \%$ y el $95 \%)^{3}$. Si bien no puede identificarse como un fenómeno exclusivo del municipio, ya que se trata de una realidad visible en toda España (Rial, Mariño y Lego, 2011; Termes, 2012; Mariño y Rial, 2019), sí que existe la necesidad de revertir esta situación. En esta línea, cuando se les pregunta a los jóvenes acerca de la FP, la mayoría considera que poseen un amplio abanico de alternativas respecto a las salidas laborales que ofrece esta opción, pero destacan e inciden en la problemática de la segregación de género de la FP:

\section{«hay ciclos de FP donde todo su alumnado es masculino y también ocurre al contrario [...] hay chicas que queriendo, no entran al ciclo de informática porque todo lo que hay son chicos [...] cuando íbamos al orientador, a los que mandaban a automoción [FP] siempre era a los chicos». (TP2).}

Aunque esto responda fundamentalmente a causas estructurales que están arraigadas en la transmisión cultural de los roles de género (Subirats, 2016), se trata de un fenómeno sobre el que se debe actuar desde el ámbito local. En este sentido, el trabajo de campo realizado en los grupos focales y los talleres culminó en un conjunto de estrategias básicas para que esta realidad cambie: a) desarrollar e impulsar campañas de promoción de una FP mixta en aquellos ciclos donde predomina un solo género (durante el desarrollo del trabajo de campo se observó ampliamente en los ciclos de informática y automoción, ambos masculinizados); b) incorporar acciones de género en los procesos de orientación

3 Esta información se ha extraído de los datos proporcionados por la Dirección General de Formación Profesional y Enseñanzas de Régimen Especial, a través del Acuerdo Territorial para la Ocupación y el Desarrollo Local de Alcoy - Ontinyent - Ibi (2020). 
formativa y laboral; c) elaborar un estudio en profundidad, en colaboración con otros municipios vecinos, que recoja las diferentes causas por las que se reproduce la segregación por sexo-género en muchos ciclos formativos a nivel local, definiendo líneas estratégicas para visibilizarlo y combatirlo; y d) incorporar el problema de la segregación por sexo-género en los proyectos que están en marcha de fomento de los ciclos de Formación Profesional.

En resumen, a través de estas acciones, aquello que la juventud consultada plantea es utilizar todos los recursos que estén a disposición de la administración local para diseñar y promover iniciativas que fomenten los modelos mixtos de formación profesional y que, de esta forma, ningún joven se abstenga de realizar un ciclo formativo por estar expuestos a cierta presión social, más o menos sistemática o latente. No obstante, son conscientes de que no se trata de una problemática unidimensional que se deba abordar únicamente desde el campo socio-educativo; comprenden que la segregación sexual del mercado laboral es una factor determinante en la concatenación de situaciones de desigualdad de género y, por consiguiente, creen preciso añadir líneas estratégicas en el área de mpleo que traten de modificar y transformar los actuales modelos de segregación ocupacional que sobresalen en las profesiones vinculadas a la FP.

\subsection{La orientación socio-educativa en edades tempranas}

El momento en el que la juventud se enfrenta a la tarea de escoger cuál será su formación resulta ser también, según cuentan, el momento en el que más escucha activa sobre orientación realizan. En este contexto, la mirada se dirige principalmente a su profesorado en busca de sugerencias y directrices. La mayoría de la juventud consultada identifica las fases de transición formativa como acontecimientos que determinan el futuro de sus vidas, reconociendo que las figuras profesionales de los centros educativos tienen una gran responsabilidad. Además, subrayan la falta de comprensión que reciben por parte de algunos profesionales que se dedican a ello.

«muchas veces los orientadores son especialistas en desorientarte más que otra cosa [...] les preguntas y te dicen que te olvides de eso, que mejor otra cosa pero ¿̇por qué?? (TP2).

Si bien la mayoría de jóvenes coincide en que Ontinyent cuenta con un profesorado de calidad, este hecho no es suficiente si se desea consolidar un modelo óptimo de orientación formativa ya que, tal y como sugieren Fernández e Ibáñez (2018), las buenas prácticas no deberían recaer sobre la buena, o mala, voluntad del profesorado de turno:

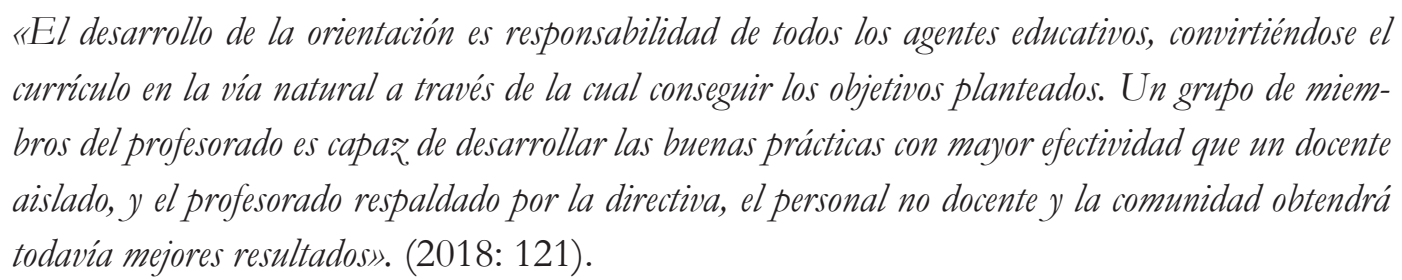

Un aspecto que también destacan, en este sentido, es que las transformaciones sociales, culturales y tecnológicas que se han producido en las últimas décadas requieren que el «reciclaje formativo» del profesorado sea considerado un imperativo. Desde el ámbito municipal, en colaboración con los diferentes centros educativos del municipio, se busca dotar a ese profesorado de las herramientas ne- 
cesarias para adaptarse a los nuevos escenarios pedagógicos y, principalmente, dotarle de los recursos oportunos para intervenir desde el aula ante las nuevas realidades y necesidades del alumnado. Por ejemplo, la coeducación de género es un método que cada vez tiene mayor aceptación en los centros de enseñanza. En este sentido, la mayoría de jóvenes se posicionan a favor de ir incorporándola progresivamente y, así, reforzar el pilar educativo hacia la igualdad de género. Este hecho mejorará su capacitación y sensibilización «aunque incidirá no solo en la equidad de género en el aula sino también en la mejora de la calidad educativa en un sentido más amplio». (Rial, Miraño y Rego, 2011: 11).

Algunos elementos que proponen como hoja de ruta para subsanar las debilidades que presencian y viven como ciudadanos y estudiantes de formación profesional hacen principalmente referencia al a) reciclaje formativo del profesorado: realizar una programación formativa dirigida a los profesionales docentes y orientadores/as formativos que se base en modelos de orientación inclusiva, y apoyar con recursos públicos los cursos de «reciclaje formativo» que se desarrollan desde las entidades públicas competentes); b) a la formación para la igualdad: realizar campañas de concienciación dirigidas a los centros locales de enseñanza (públicos y privados) donde se expongan las potencialidades de la coeducación de género; promover cursos de formación para la igualdad de género dirigidos a los y las representantes de entidades asociativas juveniles, coordinadores/as, monitores/as, docentes, u otros perfiles que estén ligados a la juventud; y c) a la comunicación y a la gestión de conflictos: proponen la necesidad de diseñar un plan formativo en materia de mediación de conflictos en las aulas dirigido a los centros educativos del municipio, y promover espacios de encuentro entre el profesorado y el alumnado donde se delibere acerca de los problemas que les afectan desde un plano de relación horizontal.

Todos estos aspectos consideran que deben estar apoyados tanto por la administración local como por organizaciones externas, con el objetivo de que exista un mínimo de recursos para iniciativas de este tipo. Asimismo, plantean que lo ideal sería construir un Plan de fomento de la Formación Profesional, como opción óptima de formación para el empleo, desde la perspectiva de la igualdad de género, el cual podría partir de la creación de una jornada anual, con la implicación de juventud del municipio y más allá de las fechas señaladas como el 8 de marzo o el 25 de noviembre, en la que se trate en profundidad el problema de la desigualdad de género en la etapa adolescente y juvenil, contando con profesionales de reconocido prestigio en la materia.

En definitiva, para vertebrar la igualdad de género, y aplicarla en los espacios sociales ocupados por la juventud, es necesario promover habilidades formativas. Aunque muchas personas se muestran sensibilizadas con dicha equidad, esta declaración no conlleva siempre actuaciones que la respeten y promuevan. Por supuesto, además de los centros educativos, existen muchas otras entidades locales -las asociaciones juveniles, por ejemplo- que, pese a contar con monitores sensibilizados con esta temática, no disponen de la formación adecuada y están limitados para diseñar actividades o acciones desde una perspectiva de género. Por tanto, debemos asumir, entre todos, la co-responsabilidad para que «la igualdad deje de ser una bonita palabra de moda y se convierta en parte intrínseca de nuestra realidad social». (Venegas, 2010: 399).

\section{Conclusiones}

A modo de conclusión, se revela que la segregación por género en el ámbito profesional-formativo atiende a nuevas tendencias emergentes de investigación, la cual la enmarcamos en tres dimensiones analíticas 
fundamentales: a) la voluntad de valorar y reivindicar la formación profesional como alternativa formativa a la Universidad; b) situar dicha discusión desde la perspectiva de género y, de este modo, contribuir desde la investigación social a los procesos de sensibilización y transformación de la desigualdad de género; y c) la irrupción de los paradigmas juvenilistas en el diseño de políticas públicas, en nuestro caso a través de mecanismos de participación.

Mediante las diferentes técnicas de investigación cualitativa se ha podido constatar un resultado sustancial: la mayoría de la juventud participante llegaba rápidamente a consensos en el proceso de deliberación sobre asuntos relacionados con la desigualdad de género. Si nos centramos en el asunto que atañe al presente artículo pudimos observar e identificar algunas intervenciones que concluían lo siguiente: existe una grave problemática en la FP debido a que el sesgo de género afecta a la mayoría de modalidades formativas ofertadas. Pero, ¿qué factores explican este escenario?, ¿qué opinión tenía la juventud al respecto?, ¿se veían capacitados para realizar propuestas viables a partir de sus competencias? Lo cierto es que cuando se les facilita espacios participativos y deliberativos surgen debates, análisis e ideas de una calidad extraordinaria.

Aunque estuvieran de acuerdo en que se suscitan múltiples problemáticas vinculadas a la FP (en ambos niveles), sus discusiones versaron sobre tres asuntos de fondo: la estigmatización de la FP, la segregación por género de la FP y la necesidad de reformular la orientación socioeducativa en las edades tempranas. En consecuencia, se pusieron sobre la mesa asuntos de carácter cultural-simbólico, como por ejemplo: el (escaso) prestigio social de la FP, la reproducción de la estigmatización de la FP en los procesos de socialización, la transmisión cultural de los roles de género desde edades tempranas y durante la etapa juvenil, y la falta de figuras femeninas o masculinas referentes en el campo de la FP feminizada o masculinizada, respectivamente. También lo relacionaron con contenido de tipo socio-laboral como: la segregación por género de las salidas profesionales, la no adaptación de las necesidades formativas al mercado laboral local, y la escasa disposición de las empresas locales para incorporar planes de igualdad. Otra dimensión a destacar fue la de tipo socio-político, ya que reflexionaron sobre las siguientes cuestiones: los intereses ocultos de los centros educativos por diversificar a su alumnado en función de las calificaciones obtenidas, las potencialidades de la administración pública local para revertir determinados focos explícitos de desigualdad de género, el reciclaje formativo del profesorado y de los y las orientadoras educativas, y, por último, las necesidades formativas de la sociedad civil en materia de igualdad de género (especialmente del tejido asociativo juvenil).

De este modo se corrobora hace patente la amplitud de miras de la juventud en asuntos que atañen, en este caso, a la desigualdad de género. Proponen un análisis que trasciende la simplificación del problema aportando críticas macro-estructurales, sirviéndose de ellas para enriquecer el debate e ir al fondo de asunto, aunque, al mismo tiempo, tratando de aterrizar en la realidad local mediante la traslación de los aspectos de tipo cultural-simbólico, socio-laboral y socio-político, al lenguaje de las políticas públicas locales. En el terreno del movimiento feminista se observa una tendencia a que la juventud (especialmente las chicas) esté adoptando un rol activo ampliamente destacable, este hecho se desarrolla en paralelo con el incremento de la sensibilización en materia de igualdad desde edades cada vez más tempranas. Sin embargo, ¿cómo afectan estos procesos de cambio social al diseño de políticas públicas juveniles desde la perspectiva de género?, ¿qué herramientas tienen a su disposición para acordar las políticas que afectan directamente a su colectivo?, ¿es la FP un asunto íntimamente ligado a la juventud? Los resultados de 
la investigación participativa concluyen que no solo es un asunto que les afecta directamente, sino que disponen de los recursos cognitivos suficientes para articular un análisis sociológico complejo con amplia sensibilidad de género.

En total se han recopilado hasta trece acciones estratégicas con competencias locales que están vinculadas, de forma explícita o implícita, a la problemática de la segregación de género de la formación profesional, las cuáles se clasifican en la siguiente tabla:

\section{Tabla IV.Relación de las trece acciones estratégicas vinculadas al Género y la FP}

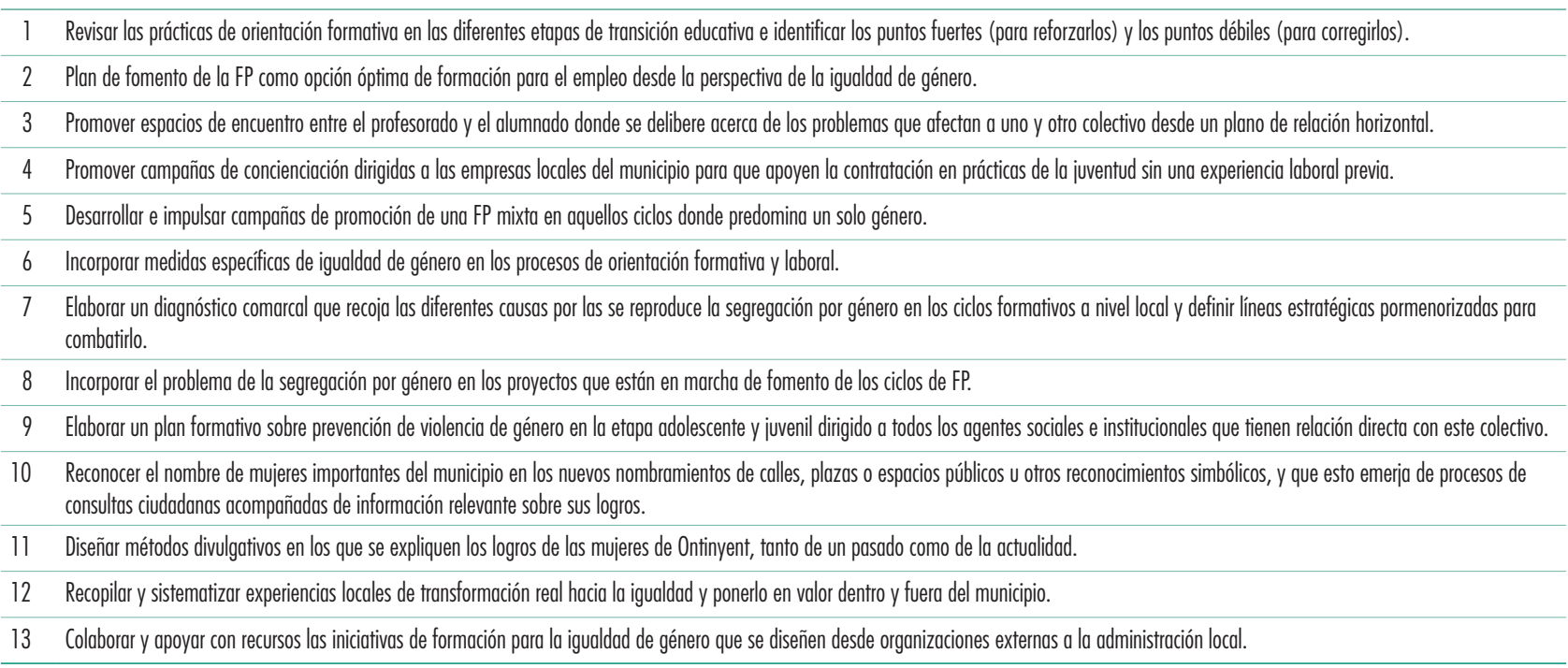

Fuente: elaboración propia.

Por tanto, la transversalidad de los debates, así como la operatividad de la técnica del flujograma, ha permitido sistematizar toda la información con el objetivo de presentar un documento tanto descriptivo como propositivo al gobierno local, y todo ello desde un denominador común: la mirada de la juventud desde un enfoque de género. La labor del equipo, como investigadores, ha sido de acompañamiento mediante una práctica de aprendizaje mutuo en la que los verdaderos logros epistémicos se han ido constituyendo durante el proceso de investigación. En este sentido, se vuelve necesario apelar a la co-responsabilidad y al compromiso de la comunidad científica en los contextos de desigualdad social y diseño de políticas públicas.

\section{Referencias bibliográficas}

Anderson, James E. (1984). Public Policy Making. An introduction. Boston: Houghton Mifflin.

Azzone, Giovanni y Dente, Bruno (1999). Valutare per governare. Milano: Etas.

Benedicto, J. y Morán, M. L. (2003). Aprendiendo a ser ciudadanos. Experiencias sociales y construcción de la ciudadanía entre los jóvenes. Madrid: INJUVE.

Brunet, Ignasi y Böcker, Rafael. (2017): "El modelo de formación profesional en España”. Revista Internacional de Organizaciones, 18, 89-108. 
Caballero, Javier; Martín, Pedro y Villasante, Tomás R. (2019): “Debatiendo las metodologías participativas. Un proceso en ocho saltos". Empiria. Revista de Metodologías de Ciencias Sociales, 44, 21-45.

Carratalá-Puertas, Liberto; Ruiz-Callado, Raúl; de-Gracia-Soriano, Pablo (2019): “La participación de la ciudadanía en los procesos de diseño de las políticas públicas de empleo. El caso del litoral sur de Alicante". Arxius de sociologia, 40, 77-90.

Colás, Pilar y Villaciervos, Patricia (2007): “La interiorización de los estereotipos de género en jóvenes y adolescentes”. Revista de Investigación Educativa, 1 (25), 35-58.

Colectivo IOE (2004). "Investigación Acción Participativa: propuesta para un ejercicio activo de la ciudadanía”. Ponencia presentada en las Jornadas sobre Movimientos Sociales e Investigación Activista. Barcelona. 22-25 de enero de 2004.

de-Gracia-Soriano, Pablo; Jareño-Ruiz, Diana; Jiménez-Delgado, María (2019): "Analysis and definition of the social, cultural and economic structure and dynamics" en Antonio Jiménez-Delgado y Jaime Lloret (eds.): Health, Wellbeing and Sustainability in the Mediterranean City: Interdisciplinary Perspectives. New York: Routledge, Taylor \& Francis.

Dye, Thomas R. (1978). Policy analysis. Alabama: University press of Alabama.

Eccles, Jacquelynne S. (2001): “Achievement” en Judith Worell (ed.): Encyclopedia of Women and Gender: Sex similarities and Differences and the Impact of Society on Gender. San Diego: Academic Press.

Fernández, Ana Belén e Ibáñez, Marta (2018): “Más mujeres en los estudios de Informática: una propuesta desde el departamento de formación y orientación laboral". RASE. Revista de Sociología de la Educación, 11 (1), 116-134.

Francés, Francisco; Alaminos, Antonio; Penalva, Clemente; Santacreu, Óscar (2015). La investigación participativa: métodos y técnicas. Ecuador: PYDLOS EDICIONES.

Ganuza, Ernesto y Francés, Francisco (2012): “The Deliberative Turn in Participation: The Problem of Inclusion and Deliberative Opportunities in Participatory Budgeting”. European Political Science Review, $4(2), 283-302$.

Goig, Juan Manuel y Núñez, María (2011): “El fomento de la juventud participativa. Tratamiento constitucional, desarrollo legislativo y políticas públicas”. Revista de Estudios de Juventud, 94, 29-48.

Hernández, Encarna; Camacho, Rafael; Silván, Aitor; Rojas-Martín, Francisco y Stan, Loredana (2018). Gobernanza participativa local. Construyendo un nuevo marco de relación con la ciudadanía. Madrid: FEMP.

Jareño-Ruiz, Diana; de-Gracia-Soriano, Pablo; Jiménez-Delgado, María (2019). "Citizen Participation in Natural and Sociocultural Landscapes Citizen Participation” en Antonio Jiménez-Delgado y Jaime Lloret (eds.): Health, Wellbeing and Sustainability in the Mediterranean City: Interdisciplinary Perspectives. New York: Routledge, Taylor \& Francis.

López, Silvia (2016): “Nuevas dimensiones en el análisis de políticas públicas. Implicaciones en el análisis de políticas de igualdad de género y juventud". Revista Metamorfosis: Revista del Centro Reina Sofía sobre Adolescencia y Juventud, 4, 22-33. 
Mariño, Raquel y Rial, Antonio (2019): "Balance de las necesidades formativas demandadas por mujeres que cursan o que han cursado ciclos de FP en familias profesionales masculinizadas: el caso de Galicia”. EDUCAR, 55 (1), 251-272.

Merino, Rafael (2012). "La formación profesional en los itinerarios formativos y laborales de los jóvenes: las leyes proponen y los jóvenes disponen". RASE. Revista de Sociología de la Educación, 5 (3), 503-512.

Mosteiro García, María Josefa y Porto Castro, Ana María (2017). "Análisis de los estereotipos de género en alumnado de formación profesional: diferencias según sexo, edad y grado”. Revista de Investigación Educativa, 35 (1), 151-165.

Ortiz, Carmen (2008): "Participación social, política y económica de la juventud española con perspectiva de género. Mujeres jóvenes hoy”. Revista de Estudios de Juventud, 83, 143-161.

Piciucchi, Danny (2019). Contribución a la elaboración de un plan estratégico para orientar las políticas destinadas a la población juvenil del municipio de Ontinyent. Alicante: Universidad de Alicante.

Rial, Antonio; Mariño, Raquel y Rego, Laura (2011): "La situación de la mujer como alumna de formación profesional inicial y como profesional en el mercado laboral”. Revista Iberoamericana de Educación, 54 (6), $1-13$.

Rosado, Ana María (2012): “Género, orientación educativa y profesional”. REMO, 9 (22), 36-41.

Sainz Ibáñez, Milagros (2007). Aspectos psicosociales de las diferencias de género en actitudes hacia las nuevas tecnologias entre los adolescentes. Madrid: INJUVE.

Subirats, Marina (2016): "De los dispositivos selectivos en la educación: el caso del sexismo". RASE. Revista de Sociología de la Educación, 9 (1), 22-36.

Termes, Andreu (2012): "La recuperación académica en la FP: alcance y potencialidades, riesgos y límites”. RASE. Revista de Sociología de la Educación, 5 (1), 58-74.

Venegas, Mar (2010). "La igualdad de género en la escuela". RASE. Revista de Sociología de la Educación, $3(3), 388-402$.

\section{Notas biográficas}

Javier Ortega es Doctor en Antropología Social por la Universidad Miguel Hernández de Elche e investigador colaborador en el Vicerrectorado de Estudiantes y Empleo, en el Departamento de Sociología I y en la Cátedra de Cultura del Pueblo Gitano de la Universidad de Alicante.

Orcid: https://orcid.org/0000-0002-5984-9477.

Danny Piciucchi es sociólogo por la Universidad de Alicante y estudiante del Máster Innovación Social y Dinámicas de Cambio en la misma universidad, en la que ha sido becario de colaboración del Departamento de Sociología I.

Orcid: https://orcid.org/0000-0001-7163-0606. 
Raúl Ruiz-Callado es Profesor Titular en el Departamento de Sociología I de la Facultad de Ciencias Económicas y Empresariales de la Universidad de Alicante e investigador asociado del Centre de Recherche en Inmigration, Ethnicité et Citoyenneté (CRIEC) de la Université du Québec à Montréal (UQÀM). Orcid: https://orcid.org/0000-0002-6784-6225.

Pablo de-Gracia-Soriano es Profesor Colaborador Honorífico de la Universidad de Alicante y Doctorando en el Programa de Doctorado de Antropología y Sociología de la Universidad Complutense de Madrid, donde es Becario FPU del Ministerio de Ciencia, Innovación y Universidades.

Orcid: https:// orcid.org/0000-0002-4809-4742. 